\title{
Fast DOA Estimation Algorithm Based on a Combination of an Orthogonal Projection and Noise Pseudo-Eigenvector Approach
}

\author{
Ching Jer Hung \\ Department of Electrical Engineering, Kun-Shan University, No. 949, Dawan Rd., Yongkang City, Tainan County 710, Taiwan
}

Correspondence should be addressed to Ching Jer Hung, andycjh@gmail.com

Received 3 November 2010; Accepted 5 December 2010

Academic Editors: C.-W. Kok, E. D. Übeyli, and Y. Xiang

Copyright () 2011 Ching Jer Hung. This is an open access article distributed under the Creative Commons Attribution License, which permits unrestricted use, distribution, and reproduction in any medium, provided the original work is properly cited.

\begin{abstract}
This paper presents a new fast direction of arrival (DOA) estimation technique, using both the projection spectrum and the eigenspectrum. First, the rough DOA range is selected using the projection spectrum; then, a linear matrix equation is used to acquire a noise pseudo-eigenvector. Finally, the fine DOA estimation is obtained from an eigenspectrum approach based on the noise pseudo-eigenvector. Without the need to form the covariance matrix from a block of the array data and without a prior knowledge of the number of incoming signals, reduced complexity is achieved, in contrast to conventional subspace-based algorithms. Simulation results show that the proposed algorithm has a good resolution performance and deals well with both uncorrelated and correlated signals. Since the new approach can reduce computational complexity while maintaining better or similar resolution capability, it may provide wider application prospects in real-time DOA estimation when contrasted to other comparable methods.
\end{abstract}

\section{Introduction}

The direction of arrival (DOA) has been applied in many fields, including speech, radio, telecommunication, and medical signal processing. In the past few decades, a large number of accurate high-resolution DOA estimation techniques have been proposed. One popular approach is the subspace-based method, used in the well-known MUltiple Signal Classification (MUSIC) algorithm [1] and Estimation of Signal Parameters by Rotational Invariance Techniques (ESPRIT) [2]. However, the conventional subspacebased method has some inherent problems that require the assumption of the uncorrelated incident signals, prior knowledge of the number of incoming signals, a block of data snapshots to form a covariance matrix, and an eigendecomposition computation. We know that the estimations for the covariance matrix and the number of the incoming signals, the computation for eigendecomposition, and the procedure for decorrelating the high correlated signals are computationally intensive and time-consuming. Therefore, the conventional subspace-based will remain severely limited in fast DOA estimation under a nonstationary environment if the above issues are not resolved.

A direct data domain least squares $\left(D^{3} \mathrm{LS}\right)$ approach [3-7] has recently been proposed and applied effectively in adaptive array processing. Based on the $\mathrm{D}^{3} \mathrm{LS}$ approach, methods $[8,9]$ developed a fast DOA estimation algorithm process, called the pseudo-covariance matrix technique, which estimated fast varying signals in two steps. The rough incidence angle ranges are first obtained using the bearing response; then, exact incidence angles are determined by combing the bearing response and the directional spectrum. The bearing response and the directional spectrum are obtained using a pseudo-covariance matrix, based on a single snapshot [8]. The advantage of the approach given in [8] is that the DOA can be estimated on a snapshotby-snapshot basis, without the need of prior knowledge of the number of incoming signals and without the need of the covariance matrix from a block of the array data. Kim's algorithm performs well for both uncorrelated and 
correlated signals coexisting in nonstationary environments. However, it requires the solution of a nonlinear-generalizedeigenvalue equation of a pseudo-covariance matrix, resulting in a high computational burden, and, using this method, the number of resolved signals is at most half of the number of the sensor array. In order to enhance the resolution of Kim's method, an improved pseudo-covariance matrix scheme was proposed. It was Wen's method [9] which is called forward-backward pseudo-covariance matrix. Wen's method processes more signals and obtains a better resolution than the forward-only matrix. However, it uses singular-value decomposition (SVD), requiring a greater computational burden than does Kim's method.

In this paper, an effective method is proposed for developing a fast DOA estimation algorithm when uncorrelated and correlated signals are present in highly nonstationary environments. The proposed method consists of two approaches. First, we use a suboptimal projection spectrum approach to select the rough DOA ranges of the incoming signals. This is done forming small spectrum peaks, generated by removing the signal from a given direction, instead of solving a more exact projection operator, as in Kim's and Wen's methods. The whole process reduces algorithm computational cost. Secondly, the solution of a linear-matrix equation, formed by a forward-backward data matrix, is applied as a noise pseudo-eigenvector, which performs a function similar to a noise eigenvector. Then, a fine DOA estimation can be obtained from an eigenspectrum. This approach allows us to reduce computational cost and obtain a good algorithm resolution. In contrast to Kim's and Wen's algorithms, the proposed algorithm has better or similar capability of resolution but offers lower computational complexity. Moreover, the proposed algorithm deals well with both uncorrelated and correlated signals and processes only a signal data snapshot without the use of the covariance matrix. Therefore, it may provide wider application prospects in real-time DOA estimation.

This paper is organized as follows. In Section 2, we briefly introduce the problem statement and signal model. In Section 3, we present the proposed DOA estimation algorithm. In Section 4, some simulation results and a comparison of the proposed algorithm to MUSIC, ESPRIT, Kim's, and Wen's methods are presented. Finally, conclusions are presented in Section 5.

\section{Problem Statement and Signal Model}

In the section, we prepare mathematical models and representations of signals. In what follows, the operators $[\cdot]^{T}$, $[\cdot]^{H},[\cdot]^{*}$, and $\|\cdot\|$ denote transpose, conjugate transpose, conjugate, and vector norm, respectively. $\operatorname{diag}\left[t_{1}, \ldots, t_{u}\right]$ represents a diagonal matrix with diagonal entries $t_{1}, \ldots, t_{u}$.

Our objective is to estimate the DOA from $q$ incident signals coming from a received single snapshot. Let a single data snapshot vector $\underline{\mathbf{b}}$ received from a uniform linear array with $m$-omnidirectional sensors, representing a linear combination of $q(m>q)$ narrow-band signals added with zero mean white Gaussian noise process, be given by

$$
\underline{\mathbf{b}}=\mathbf{A}(\theta) \underline{\mathbf{s}}+\underline{\mathbf{n}}=\left[\begin{array}{llll}
b_{1} & b_{2} & \cdots & b_{m}
\end{array}\right]^{T},
$$

where $\underline{\mathbf{s}}=\left[\begin{array}{llll}s_{1} & s_{2} & \ldots & s_{q}\end{array}\right]^{T}$ is a $q \times 1$ signal vector, $\underline{\mathbf{n}}$ is an $m \times 1$ noise process vector, and $\mathbf{A}(\theta)=\left[\underline{\mathbf{a}}\left(\theta_{1}\right), \underline{\mathbf{a}}\left(\theta_{2}\right), \ldots \underline{\mathbf{a}}\left(\theta_{q}\right)\right]$ represents a $m \times q$ matrix of steering vectors. The $i$ th column $\underline{\mathbf{a}}\left(\theta_{i}\right)=$ $\left[1, e^{j^{2 \pi d} / \lambda^{\sin \theta_{i}}}, \ldots, e^{j 2 \pi d / \lambda^{(m-1) \sin \theta_{i}}}\right]^{T}$ is the steering vector $s_{i}$ coming from $\theta_{i}, i=1,2, \ldots, q$. Parameter $d$ is the distance between the sensors and $\lambda$ is the wavelength of the carrier.

\section{Fast DOA Estimation of Proposed Method}

In this section, the proposed DOA estimation is derived, determining the rough and fine DOA estimation algorithms, presented in Sections 3.1 and 3.2, respectively.

3.1. Rough DOA Estimation Algorithm. In Kim's and Wen's methods, the rough incidence angle ranges are estimated by using the bearing response, requiring the use of nonlineargeneralized-eigenvalue or SVD algorithm in the different look directions. However, such processing may bring high computation complexity against real-time requirement. In the subsection, we present a simple novel DOA estimation algorithm, called the orthogonal projection technique, to estimate the rough DOA ranges from incoming signals using a single snapshot for achieving fast tracking varying signals under highly nonstationary environments. Let us define an orthogonal projection matrix as

$$
\boldsymbol{\Pi}^{\perp}\left(\theta_{n}\right)=\mathbf{I}-\underline{\mathbf{a}}{ }^{\prime}\left(\theta_{n}\right) \underline{\mathbf{a}^{\prime}}\left(\theta_{n}\right)^{H}, \quad n=1,2, \ldots, \frac{180}{\Delta},
$$

where $\theta_{n}=n \Delta \in\left[-90^{\circ}, 90^{\circ}\right]$ denotes each search angle, $\Delta$ is search angle step, and $\underline{\mathbf{a}}^{\prime}\left(\theta_{n}\right)$ is the normalized version of $\underline{\mathbf{a}}\left(\theta_{n}\right)$, determined as $\underline{\mathbf{a}}^{\prime}\left(\theta_{n}\right)=\underline{\mathbf{a}}\left(\theta_{n}\right) /\left\|\underline{\mathbf{a}}\left(\theta_{n}\right)\right\|$. Then, use (2) to form the following projected power spectrum:

$$
\mathbf{P}_{\Pi}\left(\theta_{n}\right)=\frac{1}{\left\|\boldsymbol{\Pi}^{\perp}\left(\theta_{n}\right) \underline{\mathbf{b}}\right\|} .
$$

When $\theta_{n} \neq \theta_{i}$ for $i=1,2, \ldots q$, the projected signal is given by

$$
\underline{\mathbf{b}}^{\perp}=\boldsymbol{\Pi}^{\perp}\left(\theta_{n}\right) \underline{\mathbf{b}}=\sum_{i=1}^{q} \boldsymbol{\Pi}^{\perp}\left(\theta_{n}\right) s_{i} \underline{\mathbf{a}}\left(\theta_{i}\right)+\Pi^{\perp}\left(\theta_{n}\right) \underline{\mathbf{n}} .
$$

Then, (4) is composed of all $q$ incident signals with noise added. However, when $\theta_{n}=\theta_{i}$ for $i=1,2, \ldots q$,

$$
\underline{\mathbf{b}}^{\perp}=\boldsymbol{\Pi}^{\perp}\left(\theta_{i}\right) \underline{\mathbf{b}}=\sum_{j=1: j \neq i}^{q-1} \boldsymbol{\Pi}^{\perp}\left(\theta_{i}\right) s_{j} \underline{\mathbf{a}}\left(\theta_{j}\right)+\Pi^{\perp}\left(\theta_{i}\right) \underline{\mathbf{n}} .
$$

Then, (5) is composed of $q-1$ incident signals with noise added, where the steering vector $\underline{\mathbf{a}}\left(\theta_{i}\right)$ is removed. The norm of (4) will have much greater value than the norm of (5). 


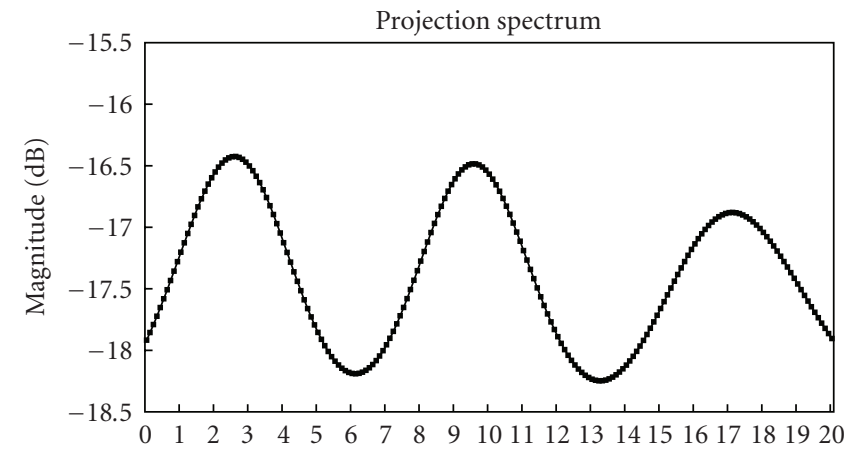

(a)

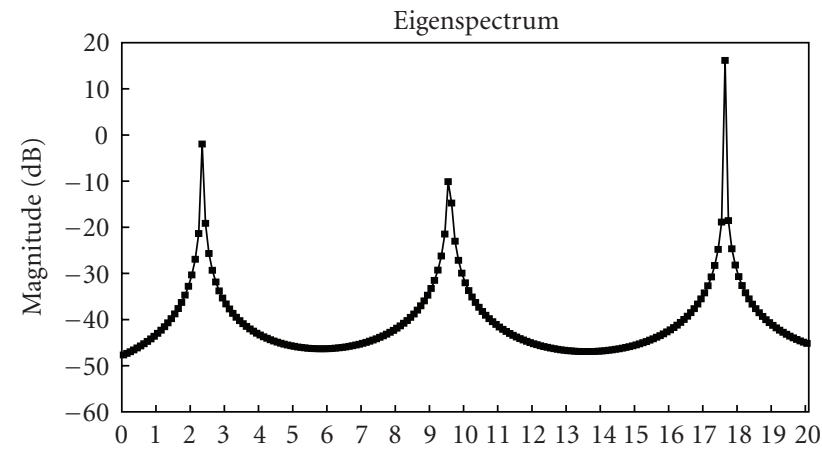

(b)

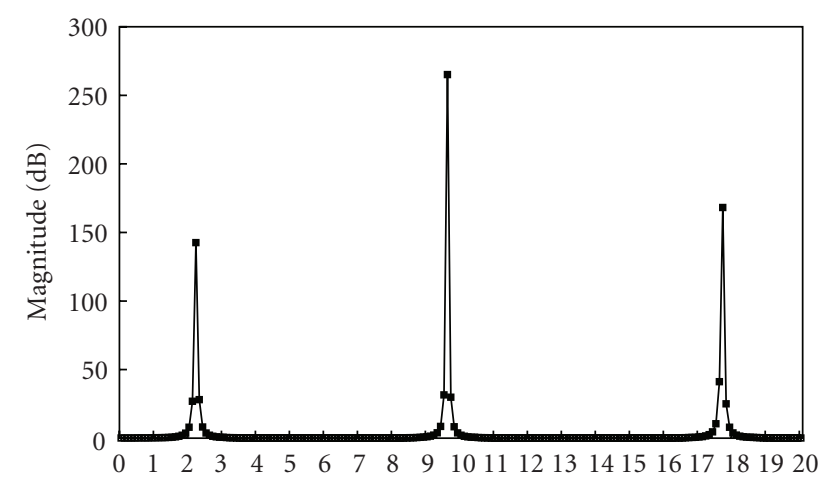

(c)

FIGURE 1: DOA estimation using the proposed algorithm and MUSIC for three uncorrelated signals from $\left[2.3^{\circ}, 9.7^{\circ}, 17.8^{\circ}\right]$ under the SNR of $20 \mathrm{~dB}$ and the number of sensors $m=23$.

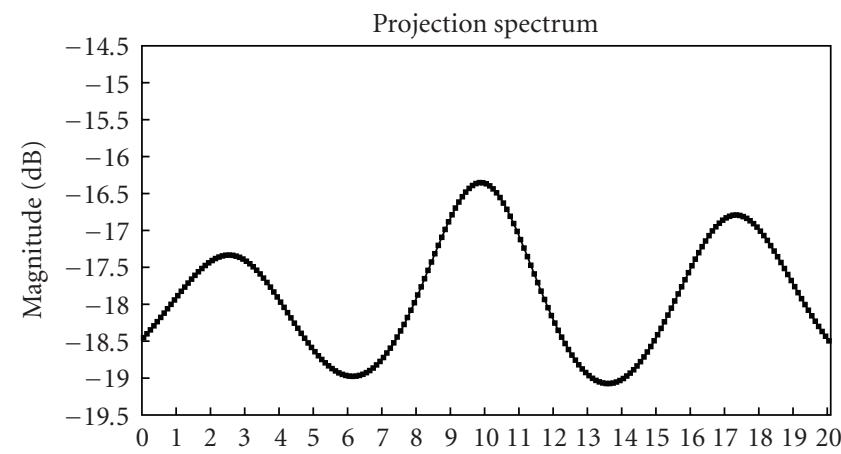

(a)

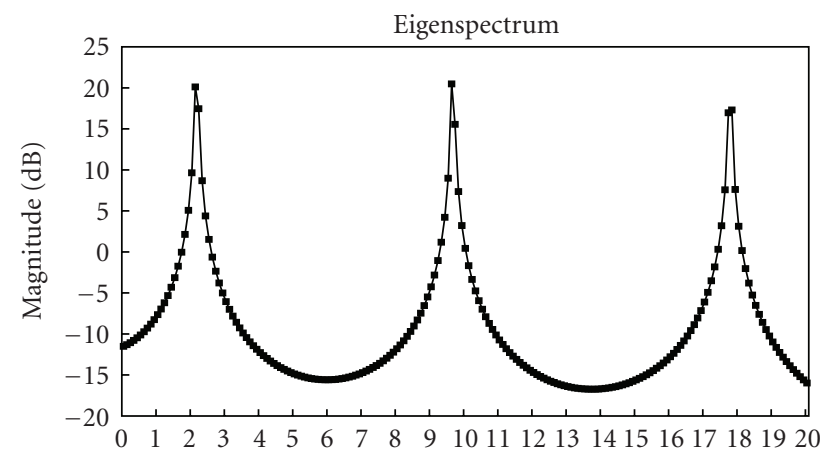

(b)

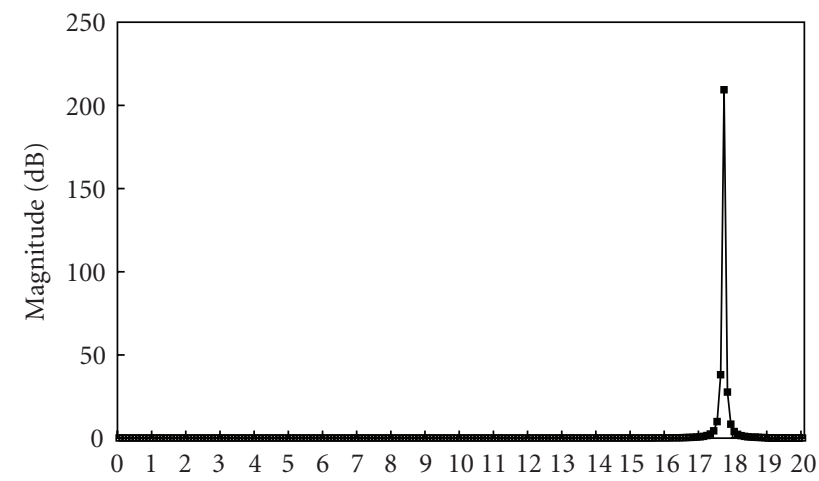

(c)

FIGURE 2: DOA estimation using the proposed algorithm and MUSIC for two coherent signals from $\left[2.3^{\circ}, 9.7^{\circ}\right]$ and one uncorrelated signal from $\left[17.8^{\circ}\right]$ under the SNR of $20 \mathrm{~dB}$ and the number of sensors $m=23$. 


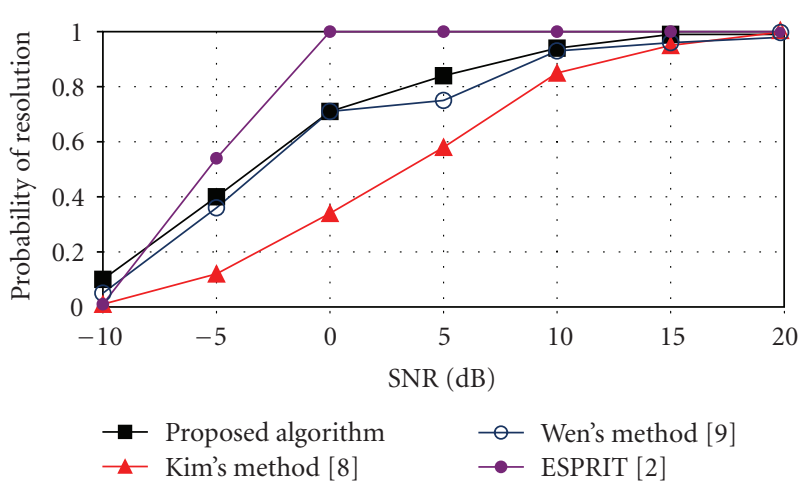

FIGURE 3: Comparison of results of the probability of resolution from ESPRIT, the proposed algorithm, Kim's, and Wen's methods, with for three uncorrelated signals from $\left[2.3^{\circ}, 9.7^{\circ}, 17.8^{\circ}\right]$ having different SNR, where the number of sensors $m=23$.

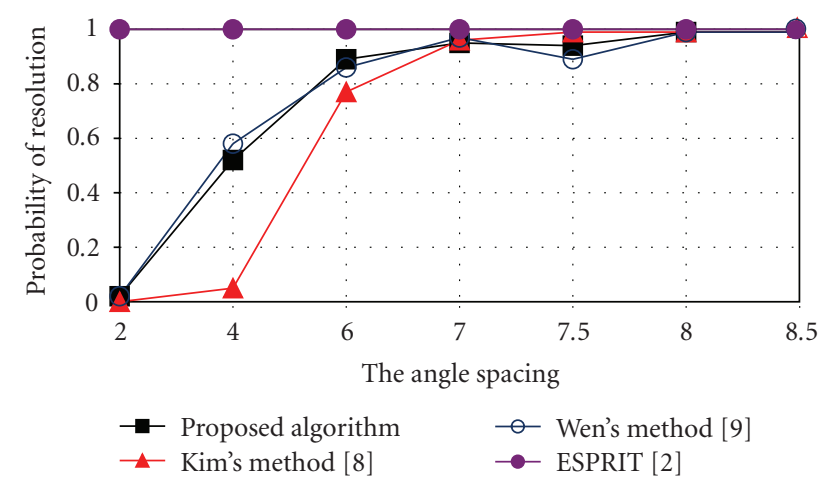

FIgURE 4: Comparison of results from ESPRIT, the proposed algorithm, Kim's, and Wen's methods, for the probability of resolution versus the angle spacing of the incoming signals for three uncorrelated signals from $\left[2.3^{\circ}, 9.7^{\circ}, 17.8^{\circ}\right]$ having the SNR of $20 \mathrm{~dB}$, where the number of sensors $m=23$.

Thus, it is easy to show that we can obtain a peak in the projected power spectrum $\mathbf{P}_{\Pi}\left(\theta_{i}\right)$ when the steering vector $\underline{\mathbf{a}}\left(\theta_{n}\right)$ coincides with the source direction, that is, $\theta_{n}=\theta_{i}$, $i=1,2, \ldots q$. By using the projection matrix $\Pi^{\perp}\left(\theta_{n}\right)$ for each steering vector $\underline{\mathbf{a}}\left(\theta_{n}\right)$, the rough DOA incident signals ranges are estimated by searching local peaks from $\mathbf{P}_{\Pi}\left(\theta_{n}\right)$, $n=1,2, \ldots, 180 / \Delta$.

3.2. Fine DOA Estimation Algorithm. In the conventional subspace-based methods, the covariance matrix is decomposed into its constituent eigenvectors and eigenvalues. Recalling that the eigenvectors of the noise eigenvalues are orthogonal to the signal subspace spanned by the eigenvectors of the signal eigenvalues, then MUSIC algorithm uses the orthogonality of these subspaces efficiently to get a highresolution DOA estimation. However, such processing is time-consuming against real-time applications and may lose performance for correlated signals. This subsection presents a procedure of finding a noise pseudo-eigenvector based on the solution of a linear-matrix equation, formed by

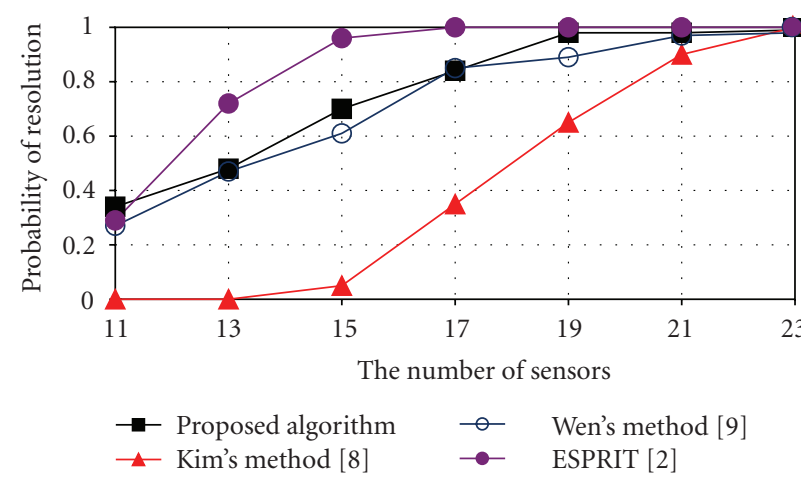

FIGURE 5: Comparison of results of results of the probability of resolution from ESPRIT, the proposed algorithm, Kim's, and Wen's methods, with three uncorrelated signals from $\left[2.3^{\circ}, 9.7^{\circ}, 17.8^{\circ}\right]$ having the different number of sensors, where the SNR of $20 \mathrm{~dB}$.

a forward-backward data matrix, for real-time requirement and for the purpose of working well in correlated signals.

By using the orthogonal projection method, we state that if $P_{\Pi}\left(\theta_{C \text { min }}\right)$ has a minimum value at angle $\theta_{n}=\theta_{C \min }$, the steering vector $\mathbf{a}\left(\theta_{C \min }\right)$ should be guaranteed to be too far from the signal directions. In order to acquire a noise pseudo-eigenvector, the steering vector $\underline{\mathbf{a}}\left(\theta_{C \min }\right)$ may be used. First, we form two data matrices, $\mathbf{B}_{1}$, and $\mathbf{B}_{2}$, by dividing the uniform linear array into $M+N$ high overlapping subarrays of size $M$ and $N$. Thus, the sensors $\{1,2, \ldots, M\}$ form the first subarray of $\mathbf{B}_{1}$ and the sensors $\{2,3, \ldots, M+1\}$ form the second subarray of $\mathbf{B}_{1}$ and so on; the sensors $\{M+2 N-1, M+2 N-2, \ldots, M+N\}$ form the first subarray of $\mathbf{B}_{2}$ and the sensors $\{M+2 N-2, M+$ $2 N-3, \ldots, M+N-1\}$ form the second subarray of $\mathbf{B}_{2}$ and so on, that is, we rearrange the received single snapshot $\underline{\mathbf{b}}=\left[\begin{array}{llll}b_{1} & b_{2} & \ldots & b_{m}\end{array}\right]^{T}$ to form the following two data matrices:

$$
\begin{gathered}
\mathbf{B}_{1}=\left[\begin{array}{cccc}
b_{1} & b_{2} & \ldots & b_{M+N} \\
b_{2} & b_{3} & \ldots & b_{M+N+1} \\
\vdots & \vdots & \ddots & \vdots \\
b_{M} & b_{M+1} & \ldots & b_{2 M+N-1}
\end{array}\right], \\
\mathbf{B}_{2}=\left[\begin{array}{cccc}
b_{M+2 N-1}{ }^{*} & b_{M+2 N-2}{ }^{*} & \ldots & b_{N}{ }^{*} \\
b_{M+2 N-2}{ }^{*} & b_{M+2 N-3}{ }^{*} & \ldots & b_{N-1} \\
\vdots & \vdots & \ddots & \vdots \\
b_{M+N}{ }^{*} & b_{M+N-1}^{*} & \ldots & b_{1}{ }^{*}
\end{array}\right],
\end{gathered}
$$

where $M$ is equal to $\lceil 2 m / 3\rceil, N(N>q)$ is equal to $\lceil(m-$ $1) / 3\rceil$, and $\lceil y\rceil(y<\lceil y\rceil)$ is the closest integer to $y$. Finally, we combine the forward data matrix (6) and the backward data matrix (7) to form an $(M+N) \times(M+N)$ forward-backward data matrix as 


$$
\begin{aligned}
\mathbf{B}_{0} & =\left[\begin{array}{lll}
\mathbf{B}_{1}{ }^{T} & \mathbf{B}_{2}{ }^{T}
\end{array}\right] \\
& =\left[\begin{array}{cccccccc}
b_{1} & b_{2} & \ldots & b_{M} & b_{M+2 N-1}{ }^{*} & b_{M+2 N-2}{ }^{*} & \ldots & b_{M+N}{ }^{*} \\
b_{2} & b_{3} & \ldots & b_{M+1} & b_{M+2 N-2}{ }^{*} & b_{M+2 N-3}{ }^{*} & \ldots & b_{M+N-1} \\
\vdots & \vdots & \ddots & \vdots & \vdots & \vdots & \ddots & \vdots \\
b_{M+N} & b_{M+N+1} & \ldots & b_{2 M+N-1} & b_{N}{ }^{*} & b_{N-1}^{*} & \ldots & b_{1}^{*}
\end{array}\right] .
\end{aligned}
$$

We know that the resolution capability of the DOA estimation is relative to the number of sensors or to the degree of freedom (DOF) of the solved data matrix $[7,9]$. For both the forward-only and backward-only methods, the maximum number of the DOF we can consider is given by $\lceil m / 2\rceil$. In the proposed algorithm, we combine the forward data matrix $\mathbf{B}_{1}$ and backward data matrix $\mathbf{B}_{2}$ to double the received data and thereby increase the DOF significantly over that of either the forward-only or backward-only method alone. The maximum number of the DOF in the forward-backward method can be increased significantly to $\lceil 2 \mathrm{~m} / 3\rceil$ without increasing the number of sensors; thus, this is the reason that we set $M=\lceil m / 3\rceil$ and $N=\lceil(m-1) / 3\rceil$. Then, the proposed algorithm with about $2 \mathrm{~m} / 3$ DOF can provide a better resolution performance than Kim's forward-only method with about $m / 2$ DOF, and a resolution performance similar to Wen's forwardbackward method with about $2 m / 3$ DOF. Let $\underline{\mathbf{b}}_{\mathrm{k}}$ denote the $k$ th column vector of the data matrix (8). Then, we can write

$$
\begin{gathered}
\underline{\mathbf{b}}_{k}=\tilde{\mathbf{A}}(\theta) \mathbf{F}^{h-1} \underline{\mathbf{s}}+\underline{\mathbf{n}}_{\mathrm{k}}, \\
(k, h)=(1,1),(2,2), \ldots,(M, M),(M+1,-M-2 N+3), \ldots,
\end{gathered}
$$$$
(M+N,-M-N+2),
$$

where $\tilde{\mathbf{A}}(\theta)$ and $\mathbf{F}$ can be expressed as

$$
\begin{aligned}
\tilde{\mathbf{A}}(\theta) & =\left[\underline{\tilde{\mathbf{a}}}\left(\theta_{1}\right), \underline{\tilde{\mathbf{a}}}\left(\theta_{2}\right), \ldots, \underline{\tilde{a}}\left(\theta_{q}\right)\right], \\
\underline{\tilde{\mathbf{a}}}\left(\theta_{i}\right) & =\left[1, e^{\varphi_{i}}, \ldots, e^{(M+N-1) \varphi_{i}}\right]^{T}, \\
\mathbf{F} & =\operatorname{diag}\left[e^{\varphi_{1}}, \ldots, e^{\varphi_{q}}\right],
\end{aligned}
$$

where $\varphi_{i}=j^{2 \pi d} / \lambda^{\sin \theta_{i}}, i=1,2, \ldots q$. Suppose that the first two signals are highly correlated or coherent among the incident signals, that is, $s_{2}=\rho s_{1}$, where $\rho$ denotes a complex coefficient describing the phase and gain relationship between the two coherent signals. Under this assumption, we can rewrite the first column vector of the data matrix (8) as

$$
\begin{aligned}
\underline{\mathbf{b}}_{1} & =\tilde{\mathbf{A}}(\theta) \mathbf{F}^{\circ} \underline{\mathbf{s}}+\underline{\mathbf{n}}_{1} \\
& =\left[\underline{\tilde{\mathbf{a}}}\left(\theta_{1}\right), \underline{\tilde{\mathbf{a}}}\left(\theta_{2}\right), \ldots, \tilde{\tilde{\mathbf{a}}}\left(\theta_{q}\right)\right]\left[\begin{array}{c}
s_{1} \\
\rho s_{1} \\
\vdots \\
s_{q}
\end{array}\right]+\underline{\mathbf{n}}_{1} \\
& =s_{1} \underline{\tilde{\mathbf{a}}}\left(\theta_{1}\right)+\rho s_{1} \underline{\tilde{\mathbf{a}}}\left(\theta_{2}\right) \cdots+s_{q} \underline{\tilde{\mathbf{a}}}\left(\theta_{q}\right)+\underline{\mathbf{n}}_{1},
\end{aligned}
$$

then

$$
\left[\underline{\mathbf{b}}_{1}-s_{1} \underline{\tilde{\mathbf{a}}}\left(\theta_{1}\right)\right]=\left[\rho s_{1} \underline{\tilde{\mathbf{a}}}\left(\theta_{2}\right)+\cdots+s_{q} \underline{\tilde{\mathbf{a}}}\left(\theta_{q}\right)\right]+\underline{\mathbf{n}}_{1} .
$$

Based on (12), $\left[\underline{\mathbf{b}}_{1}-s_{1} \underline{\tilde{\mathbf{a}}}\left(\theta_{1}\right)\right]$ excludes the desired signal $s_{1} \underline{\tilde{\mathbf{a}}}\left(\theta_{1}\right)$, that is, it only includes other $q-1$ desired signals $\rho s_{1} \underline{\tilde{\mathbf{a}}}\left(\theta_{2}\right), \ldots, s_{q} \underline{\tilde{\mathbf{a}}}\left(\theta_{q}\right)$ and noise vector $\underline{\mathbf{n}}_{1}$. If we can find a weight vector $\underline{\mathbf{w}}\left(\theta_{1}\right)=\left[w_{1}\left(\theta_{1}\right), w_{2}\left(\theta_{1}\right), \ldots, w_{M+N}\left(\theta_{1}\right)\right]^{T}$ to satisfy $\left[\rho_{1} s_{1} \underline{\tilde{\mathbf{a}}}\left(\theta_{2}\right)+\cdots+s_{q} \underline{\tilde{\mathbf{a}}}\left(\theta_{q}\right)+\underline{\mathbf{n}}_{1}\right]^{T} \underline{\mathbf{w}}\left(\theta_{1}\right)=0$, then (12) can be rewritten as

$$
\left[\underline{\mathbf{b}}_{1}-s_{1} \underline{\tilde{\mathbf{a}}}\left(\theta_{1}\right)\right]^{T} \underline{\mathbf{w}}\left(\theta_{1}\right)=0 .
$$

Therefore,

$$
\underline{\mathbf{b}}_{1}^{T} \underline{\mathbf{w}}\left(\theta_{1}\right)=s_{1} \underline{\tilde{\mathbf{a}}}\left(\theta_{1}\right)^{T} \underline{\mathbf{w}}\left(\theta_{1}\right) .
$$

By the same process, we can then get

$$
\begin{aligned}
& \underline{\mathbf{b}}_{2}^{T} \underline{\mathbf{w}}\left(\theta_{1}\right)=s_{1} e^{\varphi_{i}} \underline{\tilde{\mathbf{a}}}\left(\theta_{1}\right)^{T} \underline{\mathbf{w}}\left(\theta_{1}\right), \\
& \underline{\mathbf{b}}_{3}^{T} \underline{\mathbf{w}}\left(\theta_{1}\right)=s_{1} e^{2 \varphi_{1}} \underline{\tilde{\mathbf{a}}}\left(\theta_{1}\right)^{T} \underline{\mathbf{w}}\left(\theta_{1}\right), \\
& \text { : } \\
& \underline{\mathbf{b}}_{M}^{T} \underline{\mathbf{w}}\left(\theta_{1}\right)=s_{1} e^{(M-1) \varphi_{1}} \underline{\tilde{\mathbf{a}}}\left(\theta_{1}\right)^{T} \underline{\mathbf{w}}\left(\theta_{1}\right), \\
& \underline{\mathbf{b}}_{M+1}^{T} \underline{\mathbf{w}}\left(\theta_{1}\right)=s_{1} e^{(-M-2 N+2) \varphi_{1}} \underline{\tilde{\mathbf{a}}}\left(\theta_{1}\right)^{T} \underline{\mathbf{w}}\left(\theta_{1}\right), \\
& \underline{\mathbf{b}}_{M+2}^{T} \underline{\mathbf{w}}\left(\theta_{1}\right)=s_{1} e^{(-M-2 N+3) \varphi_{1}} \underline{\tilde{\mathbf{a}}}\left(\theta_{1}\right)^{T} \underline{\mathbf{w}}\left(\theta_{1}\right), \\
& \underline{\mathbf{b}}_{M+N}^{T} \underline{\mathbf{w}}\left(\theta_{1}\right)=s_{1} e^{(-M-N+1) \varphi_{1}} \underline{\tilde{\mathbf{a}}}\left(\theta_{1}\right)^{T} \underline{\mathbf{w}}\left(\theta_{1}\right) \text {. }
\end{aligned}
$$


Combining (12-1) and (12-2) into matrix notation gives

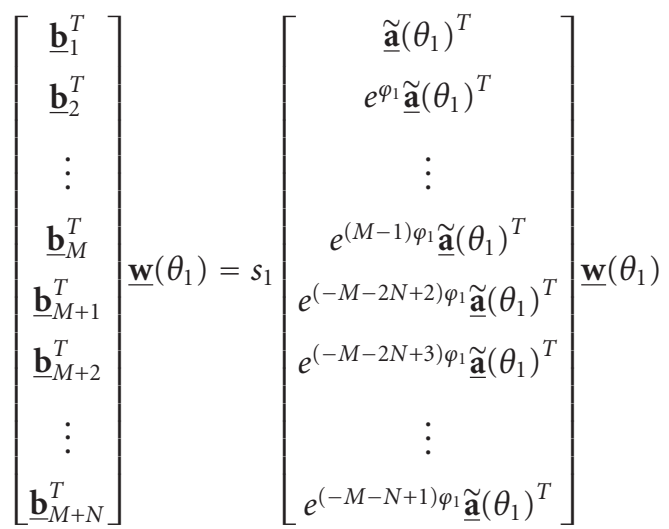

$$
\begin{aligned}
& =\left[\begin{array}{c}
s_{1} \underline{\tilde{\mathbf{a}}}\left(\theta_{1}\right)^{T} \\
s_{1} e^{\varphi_{1}} \underline{\tilde{\mathbf{a}}}\left(\theta_{1}\right)^{T} \\
\vdots \\
s_{1} e^{(M-1) \varphi_{1}} \underline{\tilde{\mathbf{a}}}\left(\theta_{1}\right)^{T} \\
s_{1} e^{(-M-2 N+2) \varphi_{1}} \underline{\underline{\mathbf{a}}\left(\theta_{1}\right)^{T}} \\
s_{1} e^{(-M-2 N+3) \varphi_{1}} \underline{\tilde{\mathbf{a}}}\left(\theta_{1}\right)^{T} \\
\vdots \\
s_{1} e^{(-M-N+1) \varphi_{1}} \underline{\tilde{\mathbf{a}}\left(\theta_{1}\right)^{T}}
\end{array}\right]\left[\begin{array}{c}
w_{1}\left(\theta_{1}\right) \\
w_{2}\left(\theta_{1}\right) \\
\vdots \\
w_{M+N}\left(\theta_{1}\right)
\end{array}\right] \\
& =s_{1} w_{1}\left(\theta_{1}\right) \underline{\hat{\tilde{a}}}\left(\theta_{1}\right)+\cdots+s_{1} w_{M+N}\left(\theta_{1}\right) \underline{\hat{\tilde{a}}}\left(\theta_{1}\right) \\
& =\alpha \underline{\hat{\tilde{a}}}\left(\theta_{1}\right),
\end{aligned}
$$

where

$$
\begin{gathered}
\alpha=\left(s_{1} w_{1}\left(\theta_{1}\right)+\cdots+s_{1} w_{M+N}\left(\theta_{1}\right)\right), \\
\underline{\hat{\mathbf{a}}}\left(\theta_{1}\right)=\left[1, e^{\varphi_{1}}, \ldots, e^{(M-1) \varphi_{1}}, e^{(-M-2 N+2) \varphi_{1}}, \ldots, e^{(-M-N+1) \varphi_{1}}\right]^{T},
\end{gathered}
$$

then

$$
\left[\begin{array}{c}
\underline{\mathbf{b}}_{1}^{T} \\
\underline{\mathbf{b}}_{2}^{T} \\
\vdots \\
\underline{\mathbf{b}}_{M}^{T} \\
\underline{\mathbf{b}}_{M+1}^{T} \\
\underline{\mathbf{b}}_{M+2}^{T} \\
\vdots \\
\underline{\mathbf{b}}_{M+N}^{T}
\end{array}\right] \underline{\mathbf{w}}\left(\theta_{1}\right) \approx \alpha \underline{\tilde{\mathbf{a}}}\left(\theta_{1}\right) \equiv \underline{\tilde{\mathbf{a}}}\left(\theta_{1}\right) \text {. }
$$

Note that (16) skips the scalar $\alpha$ because it does not affect the vector direction of weight vector $\underline{\mathbf{w}}\left(\theta_{1}\right)$. Weight vector $\underline{\mathbf{w}}\left(\theta_{1}\right)$ has pattern nulls at the incident angles of the rest of incoming signals, $\theta_{2}, \theta_{3}, \ldots, \theta_{q}$. Therefore, if the steering vector $\underline{\hat{\mathbf{a}}}\left(\theta_{C \min }\right)$ is used in (16), then the corresponding weight vector $\underline{\mathbf{w}}\left(\theta_{C \min }\right)$ will be orthogonal to the signal steering vectors $\underline{\tilde{\mathbf{a}}}\left(\theta_{i}\right)$ of the incoming signals, that is, $\underline{\mathbf{w}}\left(\theta_{C \min }\right)^{T} \underline{\tilde{\mathbf{a}}}\left(\theta_{i}\right) \approx 0$, where $i=1,2, \ldots, q$. In this paper, weight vector $\underline{\mathbf{w}}\left(\theta_{C \mathrm{~min}}\right)$ is a noise pseudo-eigenvector because it performs a function similar to that of a noise eigenvector. Then, fine peaks corresponding to the DOA of the incoming signals can be found in eigenspectrum $P_{E 1}\left(\theta_{n}\right)$ using

$$
P_{E 1}\left(\theta_{n}\right)=\frac{1}{\left|\underline{\mathbf{w}}\left(\theta_{C \min }\right)^{T} \underline{\mathbf{a}}\left(\theta_{n}\right)\right|^{2}} .
$$

Moreover, since an eigenspectrum provides higher resolution DOA estimation than a projection spectrum, steering vector $\underline{\widehat{\mathbf{a}}}\left(\theta_{E \text { min }}\right)$ with minimum eigenspectrum $P_{E 1}\left(\theta_{E \min }\right)$ via $(17)$ is more likely to be constrained too far from the signal steering vectors $\underline{\tilde{a}}\left(\theta_{i}\right)$ than steering vector $\underline{\widetilde{\mathbf{a}}}\left(\theta_{C \min }\right)$. The corresponding weight vector $\underline{\mathbf{w}}\left(\theta_{E \min }\right)$ is obtained by using $\underline{\hat{\mathbf{a}}}\left(\theta_{E \min }\right)$ in (16), which is more orthogonal to the signal steering vectors $\underline{\widetilde{a}}\left(\theta_{i}\right)$ than the weight vector $\underline{\mathbf{w}}\left(\theta_{C \min }\right)$. Then, we can obtain a more accurate DOA estimation of the incoming signals than (17) by performing an eigenspectrum as follows:

$$
P_{E 2}\left(\theta_{n}\right)=\frac{1}{\left|\underline{\mathbf{w}}\left(\theta_{E \min }\right)^{T} \underline{\underline{\mathbf{a}}}\left(\theta_{n}\right)\right|^{2}} .
$$

It is known that a linear matrix equation can be solved very efficiently by applying a conjugate gradient (CG) algorithm. The conjugate gradient method can provide a faster rate of convergence than other gradient methods and can be implemented to operate in real-time utilizing a digital signal processing chip [7, 10]. Therefore, the use of the CG algorithm makes our proposed algorithm suitable for realtime implementation. The procedure of our proposed fast DOA estimation is as follows.

(1) Obtain the rough DOA ranges of the incoming signals and a steering vector $\underline{\hat{\tilde{a}}}\left(\theta_{C \text { min }}\right)$ by using the projection method via (3).

(2) Use the steering vector $\underline{\hat{\mathbf{a}}}\left(\theta_{C \min }\right)$ in (16) to obtain a noise pseudo-eigenvector $\underline{\mathbf{w}}\left(\theta_{C \min }\right)$.

(3) Obtain the fine DOA estimation of the incoming signals by searching for local peak values in the eigenspectrum $P_{E 1}\left(\theta_{n}\right)$ via (17) within the rough DOA ranges.

(4) Use the steering vector $\underline{\hat{\mathbf{a}}}\left(\theta_{E \min }\right)$ in (16) to obtain a noise pseudo-eigenvector $\underline{\mathbf{w}}\left(\theta_{E \min }\right)$.

(5) Fine-tune the DOA estimation of the incoming signals in step (3) by searching for local peak values of the eigenspectrum $P_{E 2}\left(\theta_{n}\right)$ via (18).

The computational cost of solving a nonlinear generalized eigenvalue algorithm is about $\mathrm{o}\left(k^{3}\right)$ flops for a $k \times k$ matrix. The computational cost of solving an SVD algorithm 
is about $\mathrm{o}\left(k r^{2}\right)$ flops for a $k \times r$ matrix. The computational cost of solving the CG algorithm is about o $\left(k^{2}\right)$ flops per iteration for a $k \times k$ matrix. The size of resolved data matrix is approximately $m / 2 \times m / 2$ in Kim's method, $2 m / 3 \times(2 m / 3+$ 2) in Wen's method and $2 m / 3 \times 2 m / 3$ in our proposed algorithm. The main computational complexity difference between the other methods and the proposed algorithm is the weight vector calculation, which requires about Lo $\left(\mathrm{m}^{3} / 8\right)$ from solving a nonlinear generalized eigenvalue algorithm in Kim's method, $L o\left(8 \mathrm{~m}^{3} / 27\right)$ from solving a SVD algorithm in Wen's method, and $2 \mathrm{Ko}_{\mathrm{o}}\left(4 \mathrm{~m}^{2} / 9\right)$ in our proposed method, which is based on the CG algorithm. A flop is defined as a complex floating-point multiplication operation, where $\mathrm{o}(\cdot)$ denotes "the order of $. ", m$ is the number of the sensors, $L$ denotes the total scanning number in the possible DOA range (i.e., the $L$ different looking directions), and $K$ is the number of iterations for executing the CG algorithm. We can see that the computational complexity of the DOA estimation for fast varying signals for our proposed method is significantly smaller than those that of Kim's, and Wen's methods because $L \gg m>K \gg 2$ in general.

\section{Computer Simulation}

To compare the performance of the described DOA finding method, MUSIC, ESPRIT, Wen's, and Kim's method, in terms of the probability of resolution, the methods were investigated in the narrowband farfield DOA estimations with computer experiments. The probability of resolution is defined as follows. Two signals with DOA $\theta_{1}$ and $\theta_{2}$ are said to be resolved if the respective estimates $\hat{\theta}_{1}$ and $\hat{\theta}_{2}$ are such that both $\left|\hat{\theta}_{1}-\theta_{1}\right|$ and $\left|\hat{\theta}_{2}-\theta_{2}\right|$ are less than $\left|\theta_{1}-\theta_{2}\right| / 2$ [11]. Four different experiments were done with variations in SNR value, the separation between sources, and the number of sensors.

In each experiment, it was assumed that the powers of all the incoming sources were equal. The noise at each array sensor was assumed to be additive white Gaussian noise process with zero mean. We also assumed a uniform linear array with half-wavelength element spacing. We examined the conventional MUSIC and ESPRIT algorithm when the number of snapshots was 100, whereas the proposed algorithm, Kim's, and Wen's methods only used a single snapshot. A total of 300 independent test runs were done to get each simulated point, and the DOA scanning was performed over $\left[0^{\circ}, 90^{\circ}\right]$ with a step size of $\Delta=0.1$. In these simulations, the solution of a linear matrix equation was solved using the CG method.

In the first experiment, three signals with the same SNR of $20 \mathrm{~dB}$ were impinging on the array of 23 sensors. Figures 1(b) and 1(c) show that we can estimate the three uncorrelated signals from $\left[2.3^{\circ}, 9.7^{\circ}, 17.8^{\circ}\right]$ using the proposed algorithm and MUSIC, respectively. Figure 2(b) shows that we can still estimate the two coherent signals from $\left[2.3^{\circ}, 9.7^{\circ}\right]$ and one uncorrelated signal from $\left[17.8^{\circ}\right]$ using the proposed algorithm but Figure 2(c) shows that MUSIC was unable to estimate the DOA of the two coherent signals. Also, Figures 1(a) and 2(a) show that the angles $\left[2.3^{\circ}, 9.7^{\circ}, 17.8^{\circ}\right]$ of the three incident signals were within the estimated possible ranges.

In the second experiment, we considered three uncorrelated signals from $\left[2.3^{\circ}, 9.7^{\circ}, 17.8^{\circ}\right]$, impinging on the array of 23 sensors. Figure 3 shows the comparison result of the probability of resolution versus various SNR values. As seen, ESPRIT has best resolution, the proposed algorithm and Wen's method have almost identical resolution, and Kim's method has worst resolution. When an $\mathrm{SNR}=-5 \mathrm{~dB}$, the resolution probability of the proposed algorithm, Kim's and Wen's methods was less than $50 \%$ while it is close to $60 \%$ for ESPRIT.

In the third experiment, three uncorrelated signals from $\left[2.3^{\circ}, 2.3^{\circ}+\delta \theta, 2.3^{\circ}+2 \delta \theta\right]$, impinging on the array of 23 sensors and SNR of $20 \mathrm{~dB}$, were considered where the $\delta \theta$ denote a small angle spacing. Figure 4 shows the comparison result of the probability of resolution versus the angle spacing of the incoming signals. As seen in that figure, ESPRIT achieves a higher rate of successful source separation in comparison with the proposed algorithm, Kim's, and Wen's methods. For an angle spacing $\delta \theta=2^{\circ}$, the resolution probability of the proposed algorithm, Kim's and Wen's methods was close to $0 \%$. When an angle spacing $\delta \theta<7^{\circ}$, the Wen's method and the proposed algorithm had better performance than Kim's method.

In the fourth experiment, we considered three uncorrelated signals from $\left[2.3^{\circ}, 9.7^{\circ}, 17.8^{\circ}\right]$, impinging on the array with SNR of $20 \mathrm{~dB}$. Figure 5 shows the comparison result of the probability of resolution versus the number of sensors for ESPRIT, the proposed algorithm, Kim's, and Wen's methods. The simulations showed that the performance could be improved by using more number of sensors for all algorithms, but that more processing time is required.

As expected, from Figure 1 to Figure 5, the simulations showed that the proposed method outperformed Kim's methods and performed almost the same as Wen's method with regards to resolution capabilities because the effective array size in both the proposed algorithm and Wen's method is 15 , but in Kim's method is 11. If the number of signal sources is known exactly and has enough input data snapshots, of course, the MUSIC or ESPRIT provides us with better performance than the proposed algorithm in cases with uncorrelated signals.

\section{Conclusion}

A new fast direction of arrival estimation technique is presented based on both a simple projection spectrum and the eigenspectrum. The advantages of this method over MUSIC, ESPRIT, Kim's, and Wen's methods are as follows: (1) it has a lower computation loads; (2) it allows arbitrary signal statistics, for example, nonstationary and coherent; (3) it is capable of tracking high varying signals. Computer simulations have shown that the proposed algorithm handles the coherent or uncorrelated signals well, whereas MUSIC does not, as well as better resolution as compared with Kim's method, or similar resolution to Wen's method. Since this 
new approach improves performance by reducing computational complexity while maintaining sufficient resolution in highly nonstationary environments, it will have a wider range of prospective applications in real-time DOA estimation than other comparable methods.

\section{References}

[1] R. O. Schmidt, "Multiple emitter location and signal parameter estimation," IEEE Transactions on Antennas and Propagation, vol. 34, no. 3, pp. 276-280, 1986.

[2] A. Paulraj, A. Roy, and T. Kailath, "Estimation of signal parameters via rotational invariance techniques-esprit," in Proceedings of the 19th Asilomar Conference on Circuits, Systems and Computers, pp. 83-89, November 1985.

[3] W. Choi, T. K. Sarkar, H. Wang, and E. L. Mokole, "Adaptive processing using real weights based on a direct data domain least squares approach," IEEE Transactions on Antennas and Propagation, vol. 54, no. 1, pp. 182-190, 2006.

[4] K. Kim, T. K. Sarkar, H. Wang, and M. Salazar-Palma, "Direction of arrival estimation based on temporal and spatial processing using a direct data domain (D) approach," IEEE Transactions on Antennas and Propagation, vol. 52, no. 2, pp. 533-541, 2004.

[5] K. Kim, T. K. Sarkar, and M. S. Palma, "Adaptive processing using a single snapshot for a nonuniformly spaced array in the presence of mutual coupling and near-field scatterers," IEEE Transactions on Antennas and Propagation, vol. 50, no. 5, pp. 582-590, 2002.

[6] T. K. Sarkar, J. Koh, R. Adve et al., "A pragmatic approach to adaptive antennas," IEEE Antennas and Propagation Magazine, vol. 42 , no. 2, pp. 39-55, 2000.

[7] T. K. Sarkar et al., Smart Antennas, IEEE Press, New York, NY, USA; Wiley-Interscience, Hoboken, NJ, USA, 2003.

[8] J. T. Kim, S. H. Moon, D. S. Han, and M. J. Cho, "Fast DOA estimation algorithm using pseudocovariance matrix," IEEE Transactions on Antennas and Propagation, vol. 53, no. 4, pp. 1346-1351, 2005.

[9] Z. Wen, L.-P. Li, and P. Wei, "Fast direction finding using modified pseudocovariance matrix," IEEE Transactions on Antennas and Propagation, vol. 54, no. 12, pp. 3914-3918, 2006.

[10] G. H. Golub and C. F. Van Loan, Matrix Computations, Johns Hopkins University Press, Baltimore, Md, USA, 1983.

[11] R. Grover, D. A. Pados, and M. J. Medley, "Subspace direction finding with an auxiliary-vector basis," IEEE Transactions on Signal Processing, vol. 55, no. 2, pp. 758-763, 2007. 

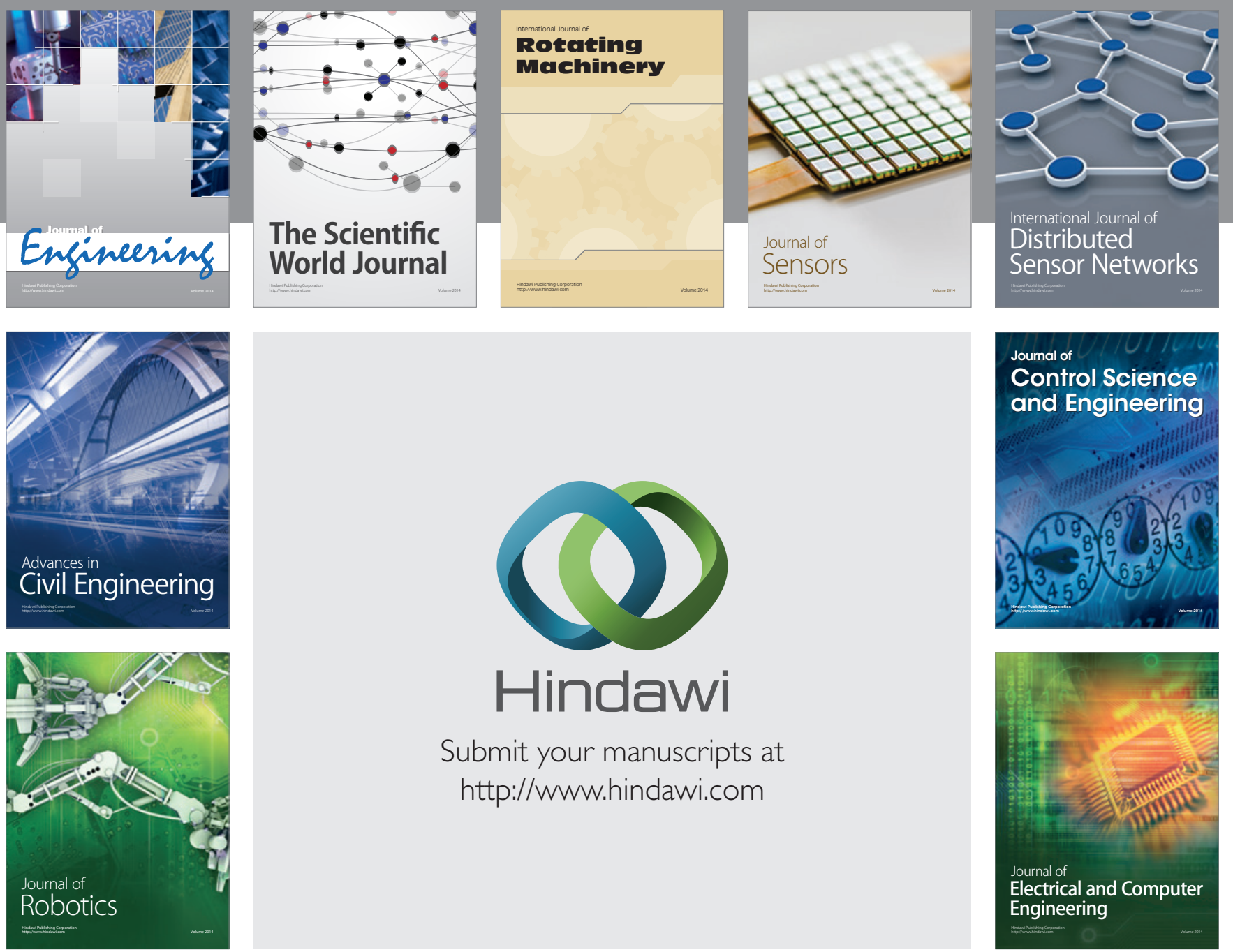

Submit your manuscripts at

http://www.hindawi.com
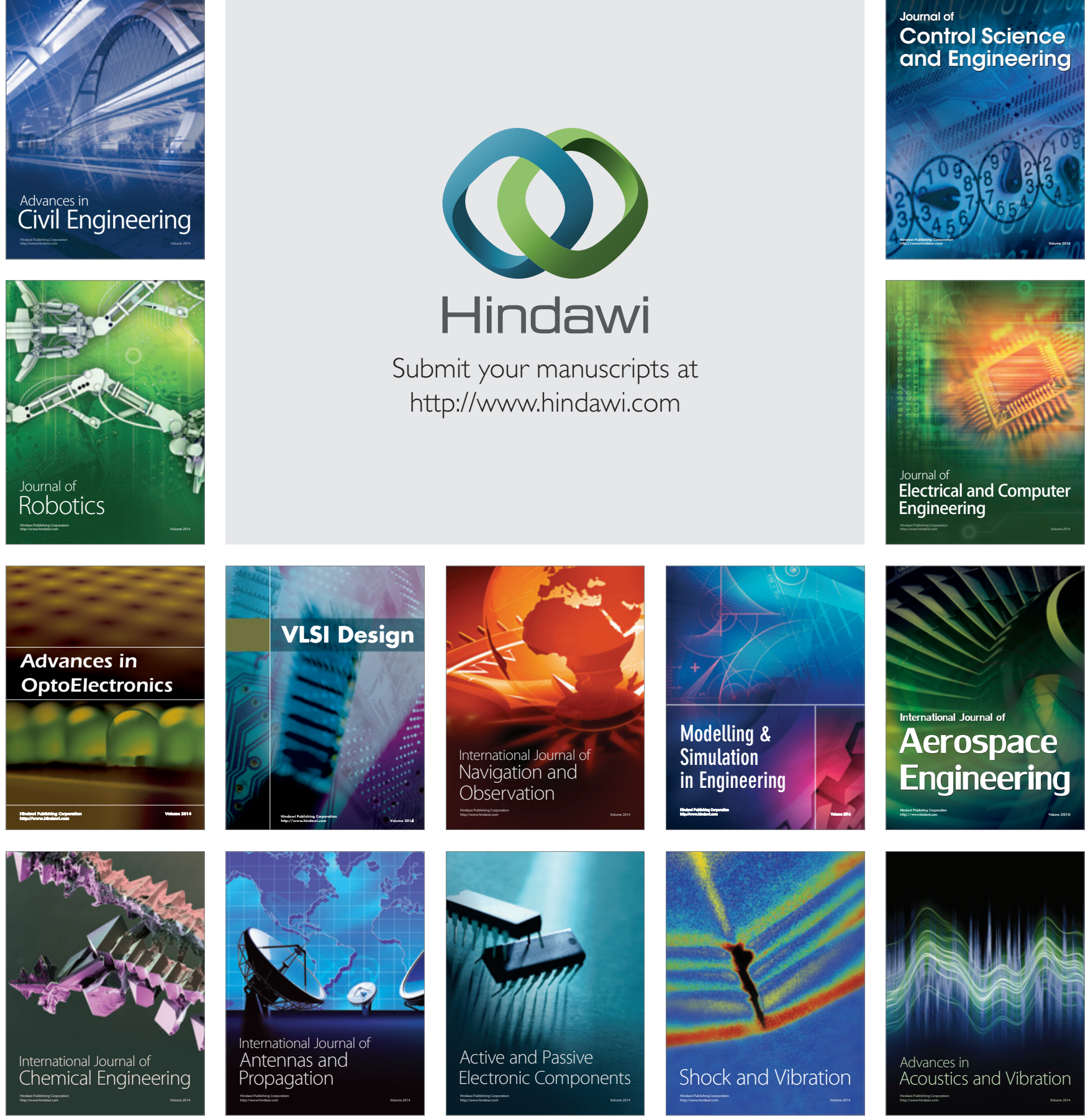